\title{
Genetic and Morphological Characterization of Colletotrichum acutatum Causing Anthracnose of Lupins
}

\author{
Pedro Talhinhas, S. Sreenivasaprasad, João Neves-Martins, and Helena Oliveira
}

First, third, and fourth authors: Instituto Superior de Agronomia, Tapada da Ajuda, 1349-017 Lisboa, Portugal; and second author: Horticulture Research International, Wellesbourne, Warwick, CV35 9EF, U.K. Accepted for publication 23 April 2002.

\begin{abstract}
Talhinhas, P., Sreenivasaprasad, S., Neves-Martins, J., and Oliveira, H. 2002. Genetic and morphological characterization of Colletotrichum acutatum causing anthracnose of lupins. Phytopathology 92:986-996.

Anthracnose, caused by Colletotrichum sp., is a serious problem of lupins (Lupinus spp.) worldwide. Morphological characters and molecular markers were used to characterize 43 Colletotrichum isolates from lupins, 8 isolates from other hosts, and 18 reference isolates representing related Colletotrichum spp., to assess the pathogen diversity and resolve its taxonomy. All lupin Colletotrichum isolates tested positive with $C$. acutatum-specific polymerase chain reaction (PCR) and did not test positive with $C$. gloeosporioides-specific PCR. Spore shape and colony diameter as well as insensitivity to benomyl grouped the lupin anthrac-

nose isolates closer to $C$. acutatum than to C. gloeosporioides. Analysis of internal transcribed spacer (ITS) sequences of 57 Colletotrichum isolates grouped all lupin isolates with $C$. acutatum and distinct from $C$. gloeosporioides. Further, tub2 and his4 sequences revealed groups concordant with ITS, reducing the excessive dependence on the latter. Arbitrarily primed-PCR and amplified fragment length polymorphism analyses revealed intraspecific subgroups, but neither was useful to decipher species level relationships. ITS, tub2, and his4 results strongly support designating lupin anthracnose pathogen as C. acutatum or its subspecies. Most Colletotrichum isolates from lupins from worldwide locations are genetically homogeneous and form a distinct subgroup within $C$. acutatum. Present results also underline the potential of the $C$. acutatum-specific PCR for routine pathogen diagnosis.
\end{abstract}

Lupinus albus (white lupin), L. angustifolius (narrow leaf lupin), and L. luteus (yellow lupin) are important crops due to their high seed protein content. Anthracnose causes typical twisting of lupin petioles and stems, with dark sunken lesions in the center of which orange conidial masses are produced. The disease was first described on L. angustifolius in the United States (48), and the causal agent was identified as Glomerella cingulata (Stoneman) Spauld. \& H. Schrenk (anamorph Colletotrichum gloeosporioides (Penz.) Penz. \& Sacc.). Anthracnose is a worldwide problem, with yield losses up to $50 \%$ on narrow leaf lupin and up to $100 \%$ on white lupin and considered the most devastating disease of lupins $(23,52)$. The taxonomy of Colletotrichum Corda isolates causing lupin anthracnose has been the subject of much debate. Based on morphology, vegetative compatibility groups (VCG), and random amplified polymorphic DNA (RAPD) data, Yang and Sweetingham (52) and Elmer et al. (13) supported the original description of $C$. gloeosporioides and identified COL1, COL2, and COL3 groups. Others suggested that these are closer to $C$. acutatum Simmonds ex Simmonds based on limited molecular and morphological data $(24,30,41)$. It has also been suggested that the lupin anthracnose pathogen is sufficiently distinct from the above two species and could be a completely different species of Colletotrichum (27).

In the genus Colletotrichum, a range of morphological and colony characters is used for species identification. For example, cylindrical conidia, and fast growing gray colonies for $C$. gloeosporioides, and fusiform conidia, and slow growing pink colonies for $C$. acutatum (44). Differential sensitivity to benomyl has also been useful in distinguishing $C$. acutatum and $C$.

Corresponding author: P. Talhinhas; E-mail address: ptalhinhas@isa.utl.pt

Publication no. P-2002-0717-02R

(c) 2002 The American Phytopathological Society gloeosporioides (1), but both have been described as group species (44). Wide variation in these characters, together with the existence of isolates exhibiting intermediate or mixed characters, make their exclusive use unreliable to distinguish these two species $(7,42,44)$. This has led to serious difficulties in reliable diagnosis of the pathogen in a number of pathosystems and implementation of control measures $(2,3,6,26)$. Nucleotide sequence of the internal transcribed spacer 1 (ITS1) of ribosomal RNA gene block (rDNA) has been used to distinguish $C$. acutatum from $C$. gloeosporioides (40) on strawberry (Fragaria $\times$ ananassa). Based on the ITS1 sequence, specific polymerase chain reaction (PCR) primers for these two species have been developed $(33,43)$. In a number of systems, in the last few years, $C$. acutatum has been identified as the causal agent instead of the widely recognized $C$. gloeosporioides $(6,7,19,32)$.

The genetic diversity within the lupin anthracnose pathogen and its relationship to $C$. gloeosporioides, $C$. acutatum, and other Colletotrichum spp. needs to be understood in order to resolve the controversy about the species identity and generate markers for accurate diagnosis of the pathogen, leading to the development of appropriate disease management and breeding strategies. The present work is based on molecular and morphological characterization of a collection of lupin anthracnose isolates with representatives from most of the lupin growing areas around the world. A range of molecular markers, namely diagnostic PCR with species-specific primers, ITS, $\beta$-tubulin 2 (tub2) and histone 4 (his4) gene-sequencing, arbitrarily primed PCR (APPCR), and amplified fragment length polymorphism (AFLP) analyses, offering different levels of resolution, have been used. We have targeted specific genes of potential taxonomic interest as well as random markers representing the genome, at both species and subspecies levels. This is the widest multilevel approach to the characterization of lupin anthracnose pathogen and the first taxonomic use of the tub2 and his 4 genes in the genus Colletotrichum. 


\section{MATERIALS AND METHODS}

Cultures used. Details of the Colletotrichum spp. isolates used in the present study are provided in Table 1. This included 43 lupin anthracnose isolates from different parts of the world and 8 Colletotrichum spp. isolates from other hosts (51 test isolates) as well as 18 previously characterized isolates representing $C$. $a c u-$ tatum (12 isolates), C. gloeosporioides (2 isolates), C. falcatum Went (2 isolates), C. graminicola (Ces.) G.W. Wils. (1 isolate), and $C$. sublineolum Henn. (1 isolate) used as reference cultures. Test isolates were isolated during 1990 to 2000, although for some the isolation date is not known. Not all isolates were used in all the analyses and further details are provided in the appropriate sections. In addition, details of a limited number of isolates for which ITS, tub2, and his 4 sequence data were downloaded from the GenBank database are also described in the results. All isolates used in this study were monoconidial cultures and are stored as agar plugs in water at room temperature at the Instituto Superior de Agronomia (Lisboa, Portugal).

Morphology and colony characters. The growth rate and colony characteristics were recorded from cultures grown on potato dextrose agar (PDA; Difco Laboratories, Detroit) plates. The cultures were incubated in darkness at a range of temperatures $(5$, $10,15,20,25,27,30,32$, and $35^{\circ} \mathrm{C}$ ), and the diameter of the colony was recorded daily (three replicates, two measurements per replicate) for 5 days. The colony characters recorded on the sixth day from cultures grown at $25^{\circ} \mathrm{C}$ in darkness were texture, density, color (38), zonation, transparency aspect, nature of the growing margin, presence of conidial masses, and color of the reverse side. For each isolate, length and width of 25 spores were measured, the length/width ratio calculated, and the shape recorded. Data were analyzed using analysis of variance and Duncan's multiple range test with Statistica software (StatSoft, Tulsa, OK). Benomyl sensitivity of colonies was analyzed on cultures grown for 5 days at $25^{\circ} \mathrm{C}$ (in darkness) on plates containing PDA amended with $2 \mathrm{mg} \mathrm{liter}^{-1}$ benomyl. Results were recorded on three replicates per isolate as the presence or absence of mycelial growth on this culture medium. A positive control was used by growing colonies on PDA without benomyl.

DNA extraction. Fungal mycelium was grown in petri dishes containing yeast extract $(0.2 \%, \mathrm{wt} / \mathrm{vol})$ and glucose $(1.0 \%, \mathrm{wt} / \mathrm{vol})$ medium (Difco) for 5 days at $25^{\circ} \mathrm{C}$. DNA was extracted from freeze-dried mycelium with the DNeasy plant kit (Qiagen, Hilden, Germany) according to manufacturer's instructions. DNA was

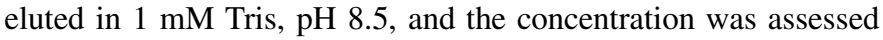
by co-electrophoresis with known quantities of $\lambda$-DNA (Life Technologies, Paisley, UK).

Diagnostic PCR with species-specific primers. CaInt2 (5'GGGGAAGCCTCTCGCGG-3'), specific for C. acutatum (43), and CgInt (5'-GGCCTCCCGCCTCCGGGCGG-3'), specific for C. gloeosporioides (33), were each used in conjunction with the conserved primer ITS4 (50). Previously characterized isolates NI90-1 and 397 (C. acutatum) and 315 (C. gloeosporioides) were used as positive controls. Amplification reaction without DNA was used as negative control.

Each PCR $(25 \mu \mathrm{l})$ contained $25 \mathrm{ng}$ of DNA, $1 \mu \mathrm{M}$ each primer, and $12.5 \mu \mathrm{l}$ of the ReadyMix RedTaq (Sigma Chemical, St. Louis). Amplifications were performed in a thermal cycler (Hybaid, Ashford, UK) programmed for 1 cycle of $5 \mathrm{~min}$ at $95^{\circ} \mathrm{C}$, 25 cycles of $30 \mathrm{~s}$ at $94^{\circ} \mathrm{C}, 30 \mathrm{~s}$ at $62^{\circ} \mathrm{C}$, and $2 \mathrm{~min}$ at $72^{\circ} \mathrm{C}$, ending with 1 cycle of $7 \mathrm{~min}$ at $72^{\circ} \mathrm{C}$. PCR products $(10 \mu \mathrm{l})$ were visualized by electrophoresis in $1.5 \%$ (wt/vol) agarose gels.

Sequencing of rDNA ITS region. Primers ITS1Ext (5'-GTAACAAGGTTTCCGTAGGTG-3') and ITS4Ext (5'-TTCTTTTCCTCCGCTTATTGATATGC-3') were used (34) for the amplification of rDNA ITS region. The reaction mix $(100 \mu \mathrm{l})$, contained $100 \mathrm{ng}$ of DNA, $1 \mu \mathrm{M}$ each primer, and $50 \mu \mathrm{l}$ of ReadyMix RedTaq. Amplification cycles were as follows: 1 cycle of $2 \mathrm{~min}$ at $95^{\circ} \mathrm{C}$,
30 cycles of $1 \mathrm{~min}$ at $94^{\circ} \mathrm{C}, 1 \mathrm{~min}$ at $55^{\circ} \mathrm{C}$, and $1 \mathrm{~min}$ at $72^{\circ} \mathrm{C}$, ending with 1 cycle of $5 \mathrm{~min}$ at $72^{\circ} \mathrm{C}$.

The amplicons (approximately $540 \mathrm{bp}$ ) were purified by gel extraction with a gel extraction kit (QIAquick; Qiagen) according to manufacturer's instructions. The resulting DNA was eluted in

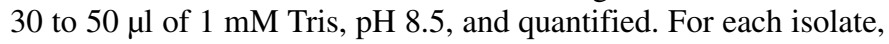
primers ITS1 and ITS4 (50) were used for sequencing with 50 to $100 \mathrm{ng}$ of the purified product. Sequencing reactions were performed by thermal cycling using a terminator cycle sequencing ready reaction kit (ABI Prism BigDye; PE Applied Biosystems, Foster City, CA). The reaction conditions and purification of the products were conducted according to the manufacturer's instructions. Electrophoresis of the fluorescent-labeled products and sequence data generation were carried out on an automated sequencer (ABI Prism 377; PE Applied Biosystems).

Sequencing variable regions in the $\boldsymbol{t u b 2}$ gene. Using published sequences of the tub2 from C. gloeosporioides (9), C. graminicola (36), and Neurospora crassa (35), four primers were designed in order to amplify and sequence variable regions of potential taxonomic interest. Primers TB5 (5'-GGTAACCAGATTGGTGCTGCCTT-3') and TB6 (5'-GCAGTCGCAGCCCTCAGCCT-3') were used for the amplification and sequencing of the variable region 1 , spanning the exons $3,4,5$, and part of 6 (36). Primers TB9 (5'AGCAGATGTTCGACCCCAAG- $3^{\prime}$ ) and TB10 (5'-CTCCTCGTCAACACCAGCGT-3') were for the variable region 2, spanning part of exon 6 and exon 7 (36). No homology was found between the primers designed for tub2 gene and published sequences of $t u b 1$ gene. No other gene sequence deposited in databases showed more homology to these primers than Colletotrichum spp. tub2.

Various steps involved in the amplification and sequencing of the two variable regions of the $t u b 2$ gene were generally the same as those described for the rDNA ITS sequencing, except for the use of $65^{\circ} \mathrm{C}$ annealing temperature in the amplification.

Partial tub2 sequences were obtained for isolates 96A4, CR02, HO01, JR03, JR15, PT29, and PT30 from lupin, isolates CR20, CR21, CR45, CR46, HO19, and JG05 from other hosts, as well as for reference isolates 397, C2897, and NI90-1 (C. acutatum), 315 (C. gloeosporioides), and CFA12 (C. falcatum).

Sequencing a variable region of his 4 gene. Part of the his4 gene was amplified using the primers and the PCR protocol developed for other filamentous fungi (22), with a $63^{\circ} \mathrm{C}$ annealing temperature. The amplicons were purified by gel extraction, quantified, and sequenced as described for ITS and tub2.

Partial his 4 sequences were obtained for isolates 546, CR02, HO01, JR03, and PT30 from lupin, isolates CR21, CR46, HO19, and JG05 from other hosts, and reference isolates 318, 397, and NI90-1 (C. acutatum), 315 (C. gloeosporioides), and 84032 (C. graminicola).

Sequence data management and analysis. ITS, tub2, and his 4 sequence files were assembled and edited to resolve ambiguities using the EditSeq and SeqMan modules in the Lasergene software package (DNAStar, Madison, WI). Consensus sequences for all isolates were compiled into a single file (Fasta format) and aligned using CLUSTAL W 1.8.1. (46). Phylogenetic analyses were performed with the PHYLIP 3.57c (University of Washington, Seattle) software package. Multiple data sets were generated by bootstrapping (SEQBOOT module) and analyzed by DNA parsimony method (DNAPARS). The neighbor-joining method (NEIGHBOR) was used to analyze distance matrices (DNADIST, Kimura-2P model) produced from the multiple data sets. For both analyses, single tree files were generated using CONSENSE and read with TreeView version 1.6.1 (available online).

Arbitrarily primed-PCR analysis. Arbitrarily primed (AP)PCR (18) was carried out using four different 15-bp primers from a total of seven tested. Primer $\mathrm{GACAC}_{3}(18)$ was used at $48^{\circ} \mathrm{C}$ annealing temperature, primer $\mathrm{CAG}_{5}$ (37) was used at $60^{\circ} \mathrm{C}$, and primers $\mathrm{TCC}_{5}$ and MR (5'-GAGGGTGGCGGTTCT-3') at $50^{\circ} \mathrm{C}(5)$. 
Amplification conditions, in general, were similar to those described for diagnostic PCR, except the use of a single primer in each reaction. Amplification cycles were adapted (21) to 1 cycle of $5 \mathrm{~min}$ at $95^{\circ} \mathrm{C}, 30$ cycles of $1 \mathrm{~min}$ at $94^{\circ} \mathrm{C}, 2 \mathrm{~min}$ at appropriate annealing temperature, and $2 \mathrm{~min}$ at $72^{\circ} \mathrm{C}$, ending with 1 cycle of $5 \mathrm{~min}$ at $72^{\circ} \mathrm{C}$. Amplification reactions were electrophoresed under uniform conditions in $1.5 \%$ agarose gels $\left(5 \mathrm{~V} \mathrm{~cm}^{-1}, 2.5 \mathrm{~h}\right)$, and the profiles were saved as electronic images for further analyses. From these image files, bands were detected and matched using the software Phoretix 1D Advanced version 4.0 (Nonlinear Dynamics, Newcastle upon Tyne, UK) and the presence or absence of bands was recorded in a binary matrix. Cluster analysis was performed by unweighted pair-group method with arithmetic average on bootstrapped distance matrices (calculated with Dice coefficient) using the program WinBoot. PHYLIP was used for generating bootstrapped matrices (SEQBOOT) and to perform parsimony analysis either with Dollo or polymorphism algorithms (DOLLOP). A consensus dendrogram was generated with CONSENSE and read with TreeView. This analysis was carried out for all test and reference isolates listed in Table 1.
AFLP analysis. The AFLP reactions (47) were carried out using the AFLP microbial fingerprinting kit (PE Applied Biosystems) according to manufacturer's instructions (DNA restriction with EcoRI and $M s e I$ ). Thirty-three isolates (test isolates from lupin [96A4, CR02, HO01, HY09, JR03, PT30, and PT40] and other hosts [CMG12, CR21, CR45, CR46, HO19, JG05, and TN47] and reference isolates for C. acutatum [287, 302a, 318, 397, 455, 473, 546, C2897, NI90-1, PD85/694, PD88/673/1, PD89/582/1, and PD90/443], C. gloeosporioides [254 and 315], C. graminicola [84032], C. falcatum [CFA5 and CFA12], and $C$. sublineolum [81607]) were analyzed with four primer combinations, two with four $(2+2)$ selective nucleotides $(\mathrm{E}-\mathrm{AA}, \mathrm{M}-\mathrm{CC}$ and E-AG, and M-CT) and two with three $(2+1$ and $1+2)$ selective nucleotides (E-AT, M-C and E-C, and M-CA). The presence or absence of bands was scored using Genescan software (PE Applied Biosystems) and recorded in a binary matrix, and data were studied by cluster and parsimony analyses as described for AP-PCR.

Nucleotide sequences. The nucleotide sequences obtained were deposited in EMBL. In cases where more than one isolate had

TABLE 1. Details of the Colletotrichum isolates used in this study and grouping based on internal transcribed spacer (ITS), tub2, his4, arbitrarily primed polymerase chain reaction (AP-PCR), amplified fragment length polymorphism (AFLP) molecular analyses

\begin{tabular}{|c|c|c|c|c|c|c|c|c|c|c|}
\hline \multirow[b]{2}{*}{ Original code } & \multirow[b]{2}{*}{ Received as } & \multirow[b]{2}{*}{ Identified as ${ }^{\mathrm{a}}$} & \multirow[b]{2}{*}{ Host } & \multirow{2}{*}{$\begin{array}{l}\text { Geographic } \\
\text { location/source }^{\text {b }}\end{array}$} & \multirow{2}{*}{$\begin{array}{l}\text { Species- } \\
\text { specific } \\
\text { PCR }^{\mathrm{c}}\end{array}$} & \multicolumn{5}{|c|}{ Grouping based on } \\
\hline & & & & & & ITS & tub2 & his4 & AP-PCR & AFLP \\
\hline \multicolumn{11}{|l|}{ Test isolates } \\
\hline G52 & C. acutatum & $\ldots$ & Lupinus albus & Germanye & $\mathrm{CA}$ & A1 & - & - & A1 & - \\
\hline PT41 & Colletotrichum & C. acutatum & Lupinus albus & Germany & CA & A1 & - & - & A1 & - \\
\hline 70354 & Colletotrichum & C. acutatum & Lupinus luteus & Germany ${ }^{\mathrm{d}}$ & $\mathrm{CA}$ & A1 & - & - & $\mathrm{A} 1$ & - \\
\hline CR02 & Colletotrichum & C. acutatum & Lupinus albus & Portugal $^{\mathrm{h}}$ & $\mathrm{CA}$ & A1 & A1 & A1 & A1 & A1 \\
\hline PT38 & Colletotrichum & C. acutatum & Lupinus albus & Portugal & $\mathrm{CA}$ & A1 & - & - & A1 & - \\
\hline PT39 & Colletotrichum & C. acutatum & Lupinus albus & Portugal & CA & A1 & - & - & $\mathrm{A} 1$ & - \\
\hline PT40 & Colletotrichum & C. acutatum & Lupinus albus & Portugal & $\mathrm{CA}$ & A1 & - & - & A1 & A1 \\
\hline IMI350308 & C. acutatum & $\ldots$ & Lupinus sp. & U.K. ${ }^{i}$ & $\mathrm{CA}$ & - & - & - & A1 & - \\
\hline $547 / 90.410$ & C. acutatum & $\ldots$ & Lupinus sp. & U.K. ${ }^{j}$ & $\mathrm{CA}$ & - & - & - & A1 & - \\
\hline 546 & C. acutatum & $\ldots$ & Lupinus sp. & U.K. ${ }^{\mathrm{j}}$ & CA & - & - & A1 & A1 & A1 \\
\hline 96A649 & C. gloeosporioides & C. acutatum & Lupinus sp. & Australia $^{1}$ & CA & A1 & - & - & A1 & - \\
\hline 70555 & Colletotrichum & C. acutatum & Lupinus albus & Chile $^{\mathrm{d}}$ & $\mathrm{CA}$ & A1 & - & - & A1 & - \\
\hline HY09 & C. gloeosporioides & C. acutatum & Lupinus albus & Canada ${ }^{1}$ & CA & A1 & - & - & A1 & A1 \\
\hline HO01 & Colletotrichum & C. acutatum & Lupinus albus & Azores, Portugal ${ }^{\mathrm{m}}$ & $\mathrm{CA}$ & A2 & A2 & A2 & A2 & A2 \\
\hline JR03 & Colletotrichum & C. acutatum & Lupinus albus & Azores, Portugal ${ }^{\mathrm{m}}$ & $\mathrm{CA}$ & A2 & A2 & A2 & A2 & A2 \\
\hline JR11 & Colletotrichum & C. acutatum & Lupinus albus & Azores, Portugal $^{\mathrm{m}}$ & $\mathrm{CA}$ & A1 & - & - & A1 & - \\
\hline \multirow[t]{2}{*}{ JR15 } & Colletotrichum & C. acutatum & Lupinus albus & Azores, Portugal ${ }^{\mathrm{m}}$ & CA & A1 & A1 & - & $\mathrm{A} 1$ & - \\
\hline & & & & & & & & \multicolumn{3}{|c|}{ (continued on next page) } \\
\hline
\end{tabular}

a ... indicates confirmation of original identity.

${ }^{\mathrm{b}}$ Source from which isolates were obtained but not necessarily the country of origin of host material.

${ }^{\text {c }}$ CA indicates positive product with $C$. acutatum-specific primer CaInt2; CG indicates positive product with $C$. gloeosporioides-specific primer $C g$ Int; and nt indicates not tested in this experiment.

${ }^{\mathrm{d}}$ H. Nirenberg, BBLF, Germany.

e J. Gondran, INRA, France.

${ }^{\mathrm{f}} \mathrm{K}$. Hughes, CSL, United Kingdom.

$\mathrm{g}$ I. Frencel, Polish Academy of Sciences, Poland.

${ }^{\mathrm{h}}$ C. Rego (isolate TN47 from T. Nascimento), LPVVA, Portugal.

${ }^{i}$ International Mycological Institute, Egham, United Kingdom.

j S. Sreenivasaprasad, HRI, United Kingdom (40-42).

${ }^{\mathrm{k}} \mathrm{S}$. Koch, ARC-PPRI, South Africa.

${ }^{1}$ H. Yang, AWA, Australia.

mJ. Rocha, University of Azores, Portugal.

${ }^{\mathrm{n}}$ C. Moura-Guedes, CEPTA, Portugal.

${ }^{\circ}$ With some markers, these isolates did not cluster with the identified subgroups A1 to A4 and group B.

p J. Chipili, HRI, United Kingdom (12).

${ }^{\mathrm{q}}$ These reference isolates were not included in the group designations with any of the markers. 
identical sequence, only one accession number was obtained representing the sequence common to those isolates. Details of other isolates with identical sequence have been provided in the EMBL data entry forms.

The rDNA ITS accession numbers are AJ300560 (isolate CR21); AJ313178 (CR45); AJ300563 (CR46); AJ311391 (HY09); AJ300557 (JG05); AJ300558 (identical sequence for isolates C2897, CR20, HO01, and JR03); AJ300559 (HO19); AJ300561 (identical sequence for isolate CMG12 and 37 other isolates; this included all isolates from lupins, except HO01, HY09, and JR03 and isolates 546, 547/90.410, and 550 sequenced previously); and AJ300562 (TN47).

For tub2 sequences, two accession numbers are given for each isolate, the first accession number is for region $1(550 \mathrm{bp})$ and the second for region 2 (488 bp): AJ409292 and AJ409291 (isolate 315); AJ409296 and AJ409295 (397); AJ314720 and AJ314719 (96A4); AJ314718 and AJ314717 (C2897); AJ409290 and AJ409289 (CFA12); AJ409298 and AJ409297 (CR02); AJ314716 and AJ314715 (CR20); AJ314714 and AJ314713 (CR21); AJ292249 and AJ292248 (CR45); AJ311668 and AJ292252 (CR46); AJ314722 and AJ314721 (HO01); AJ292241 and AJ292242 (HO19); AJ409302 and AJ409301 (JG05); AJ409300 and AJ409299 (JR03);
AJ300709 and AJ300708 (JR15); AJ409294 and AJ409293 (NI90-1); AJ314712 and AJ314711 (PT29); and AJ292250 and AJ292251 (PT30).

Accession numbers for his 4 sequences are AJ314726 (isolate 315); AJ314729 (318); AJ314731 (397); AJ314730 (546); AJ314728 (84032); AJ314725 (CR02); AJ314732 (CR21); AJ314733 (CR46); AJ314734 (HO01); AJ314735 (HO19); AJ314724 (JG05); AJ314727 (JR03); AJ314723 (NI90-1); and AJ314736 (PT30).

\section{RESULTS}

Spore morphology and colony characteristics. Spore shape revealed some interesting trends between different isolate groups (Table 2). For example, Colletotrichum isolates from Lupinus spp. and other hosts, as well as the reference $C$. acutatum isolates, had 0 to $28 \%$ round end spores, 40 to $100 \%$ one round and one acute ended spores, and 0 to $60 \%$ acute spores. However, two subgroups were observed within this collection of isolates, one with a higher proportion of acute spores (44 to 60\%) and the other with a higher proportion of intermediary spores (68 to $100 \%$ ). The first subgroup was comprised of isolates C2897 and JR03 (corresponding in part to the group of isolates producing pink colonies with

TABLE 1. (continued from preceding page)

\begin{tabular}{|c|c|c|c|c|c|c|c|c|c|c|}
\hline \multirow[b]{2}{*}{ Original code } & \multirow[b]{2}{*}{ Received as } & \multirow[b]{2}{*}{ Identified as ${ }^{\mathrm{a}}$} & \multirow[b]{2}{*}{ Host } & \multirow{2}{*}{$\begin{array}{l}\text { Geographic } \\
\text { location/source }^{b}\end{array}$} & \multirow{2}{*}{$\begin{array}{l}\text { Species- } \\
\text { specific } \\
\text { PCR }^{c}\end{array}$} & \multicolumn{5}{|c|}{ Grouping based on } \\
\hline & & & & & & ITS & $t u b 2$ & his4 & AP-PCR & AFLP \\
\hline JR16 & Colletotrichum & C. acutatum & Lupinus albus & Azores, Portugal ${ }^{\mathrm{m}}$ & $\mathrm{CA}$ & A1 & - & - & A1 & - \\
\hline JR17 & Colletotrichum & C. acutatum & Lupinus albus & Azores, Portugal & $\mathrm{CA}$ & A1 & - & - & A1 & - \\
\hline PT22 & Colletotrichum & C. acutatum & Lupinus albus & Azores, Portugal & $\mathrm{CA}$ & A1 & - & - & A1 & - \\
\hline PT23 & Colletotrichum & C. acutatum & Lupinus albus & Azores, Portugal & $\mathrm{CA}$ & A1 & - & - & A1 & - \\
\hline РT24 & Colletotrichum & C. acutatum & Lupinus albus & Azores, Portugal & $\mathrm{CA}$ & A1 & - & - & A1 & - \\
\hline PT25 & Colletotrichum & C. acutatum & Lupinus albus & Azores, Portugal & $\mathrm{CA}$ & A1 & - & - & A1 & - \\
\hline РT26 & Colletotrichum & C. acutatum & Lupinus luteus & Azores, Portugal & $\mathrm{CA}$ & A1 & - & - & A1 & - \\
\hline РT27 & Colletotrichum & C. acutatum & Lupinus luteus & Azores, Portugal & $\mathrm{CA}$ & A1 & - & - & A1 & - \\
\hline PT28 & Colletotrichum & C. acutatum & Lupinus luteus & Azores, Portugal & $\mathrm{CA}$ & A1 & - & - & A1 & - \\
\hline РT29 & Colletotrichum & C. acutatum & Lupinus albus & Azores, Portugal & $\mathrm{CA}$ & A1 & A1 & - & A1 & - \\
\hline PT30 & Colletotrichum & C. acutatum & Lupinus albus & Azores, Portugal & $\mathrm{CA}$ & A1 & A 1 & A1 & $\mathrm{A} 1$ & A1 \\
\hline PT31 & Colletotrichum & C. acutatum & Lupinus albus & Azores, Portugal & $\mathrm{CA}$ & A1 & - & - & A1 & - \\
\hline PT32 & Colletotrichum & C. acutatum & Lupinus albus & Azores, Portugal & $\mathrm{CA}$ & A1 & - & - & A1 & - \\
\hline PT33 & Colletotrichum & C. acutatum & Lupinus albus & Azores, Portugal & $\mathrm{CA}$ & A1 & - & - & A1 & - \\
\hline PT34 & Colletotrichum & C. acutatum & Lupinus luteus & Azores, Portugal & $\mathrm{CA}$ & A1 & - & - & A1 & - \\
\hline PT35 & Colletotrichum & C. acutatum & Lupinus albus & Azores, Portugal & $\mathrm{CA}$ & A1 & - & - & A1 & - \\
\hline PT36 & Colletotrichum & C. acutatum & Lupinus albus & Azores, Portugal & $\mathrm{CA}$ & A1 & - & - & A1 & - \\
\hline PT37 & Colletotrichum & C. acutatum & Lupinus albus & Azores, Portugal & $\mathrm{CA}$ & A1 & - & - & A1 & - \\
\hline JG05 & C. gloeosporioides & C. acutatum & Ceanothus sp. & France $^{\mathrm{e}}$ & $\mathrm{CA}$ & A4 & A4 & A4 & A4 & A4 \\
\hline CMG12 & Colletotrichum & C. acutatum & Cinnamonium zeylanicum & Portugal $^{\mathrm{n}}$ & $\mathrm{CA}$ & A1 & - & - & A1 & A1 \\
\hline TN47 & Colletotrichum & C. acutatum & Eriobotrya japonica & Portugal $^{\mathrm{h}}$ & $\mathrm{CA}$ & A4 & - & - & A4 & A4 \\
\hline CR20 & Colletotrichum & C. acutatum & Fragaria $\times$ ananassa & Portugal $^{\mathrm{h}}$ & $\mathrm{CA}$ & $\mathrm{A} 2$ & A2 & - & $-^{\mathrm{o}}$ & - \\
\hline CR46 & Colletotrichum & C. acutatum & Vitis vinifera & Portugal $^{\mathrm{h}}$ & $\mathrm{CA}$ & A3 & A3 & A3 & A3 & A3 \\
\hline HO19 & Colletotrichum & C. gloeosporioides & Citrus sp. & Portugal & $\mathrm{CG}$ & $\mathrm{B}$ & B & B & $-^{\mathrm{o}}$ & $\mathrm{B}$ \\
\hline CR21 & Colletotrichum & C. gloeosporioides & Citrus sp. & Portugal $^{\mathrm{h}}$ & CG & B & B & B & $-^{\mathrm{o}}$ & B \\
\hline CR45 & Colletotrichum & C. gloeosporioides & Citrus sp. & Portugal $^{\mathrm{h}}$ & $\mathrm{CG}$ & B & B & - & $-^{\mathrm{o}}$ & B \\
\hline \multicolumn{11}{|c|}{ Reference isolates } \\
\hline NI90-1 & C. acutatum & $\ldots$ & Fragaria $\times$ ananassa & U.K. ${ }^{j}$ & $\mathrm{CA}$ & A4 & A4 & A4 & A4 & A4 \\
\hline 397 & C. acutatum & $\ldots$ & Fragaria $\times$ ananassa & U.S.A.j & $\mathrm{CA}$ & $\mathrm{A} 2$ & $\mathrm{~A} 2$ & A & A2 & A2 \\
\hline PD88/673/1 & C. acutatum & $\ldots$ & Anemone sp. & Holland ${ }^{j}$ & nt & - & - & - & $-^{\mathrm{o}}$ & $-^{\mathrm{o}}$ \\
\hline PD85/694 & C. acutatum & $\ldots$ & Chrysanthemum sp. & Holland $^{\mathrm{j}}$ & $\mathrm{nt}$ & - & - & - & $-^{\mathrm{o}}$ & $-^{\mathrm{o}}$ \\
\hline PD89/582/1 & C. acutatum & $\ldots$ & Cyclamen sp. & Holland ${ }^{\mathrm{j}}$ & nt & - & - & - & $-^{\mathrm{o}}$ & $-^{\circ}$ \\
\hline C2897 & C. acutatum & $\ldots$ & Fragaria $\times$ ananassa & Australial $^{\mathrm{l}}$ & $\mathrm{CA}$ & $\mathrm{A} 2$ & A2 & - & $\mathrm{A} 2$ & A2 \\
\hline PD90/443 & C. acutatum & $\ldots$ & Phlox sp. & Holland ${ }^{j}$ & nt & - & - & - & $-^{0}$ & $-^{\mathrm{o}}$ \\
\hline 473 & C. acutatum & $\ldots$ & Liriodendron tulipifera & U.K. ${ }^{j}$ & nt & - & - & - & A3 & A3 \\
\hline 318 & C. acutatum & $\ldots$ & Magnolia sp. & U.K. ${ }^{j}$ & $\mathrm{nt}$ & - & - & A3 & A3 & A3 \\
\hline $302 \mathrm{a}$ & C. acutatum & $\ldots$ & Nandina sp. & U.K. ${ }^{j}$ & nt & - & - & - & A3 & A3 \\
\hline 455 & C. acutatum & $\ldots$ & Photinia sp. & U.K. ${ }^{j}$ & nt & - & - & - & $-^{0}$ & $-^{\mathrm{o}}$ \\
\hline 287 & C. acutatum & $\ldots$ & Statice sp. & U.K. ${ }^{\mathrm{j}}$ & nt & - & - & - & $-^{\mathrm{o}}$ & $-^{\mathrm{o}}$ \\
\hline 254 & C. gloeosporioides & $\ldots$ & Fragaria $\times$ ananassa & Canada $^{j}$ & & - & - & - & $-^{\mathrm{o}}$ & $\mathrm{B}$ \\
\hline 315 & C. gloeosporioides & $\ldots$ & Fragaria $\times$ ananassa & U.S.A.j & $\mathrm{CG}$ & B & $\mathrm{B}$ & B & $-^{\mathrm{o}}$ & $\mathrm{B}$ \\
\hline CFA12 & C. falcatum & $\ldots$ & Saccharum officinarum & India $^{p}$ & nt & $-\mathrm{q}$ & $-\mathrm{q}$ & - & $-q$ & $-\mathrm{q}$ \\
\hline CFA5 & C. falcatum & $\ldots$ & Saccharum officinarum & $\operatorname{India}^{p}$ & $\mathrm{nt}$ & - & - & - & $-q$ & $-\mathrm{q}$ \\
\hline 84032 & C. graminicola & $\ldots$ & Zea mays & Zimbabwe $^{j}$ & $\mathrm{nt}$ & - & - & $-\mathrm{q}$ & $-q$ & $-q$ \\
\hline 81607 & C. sublineolum & $\ldots$ & Apium graveolens & Australia ${ }^{\mathrm{j}}$ & $\mathrm{nt}$ & - & - & - & $-\mathrm{q}$ & $-\mathrm{q}$ \\
\hline
\end{tabular}


abundant orange spore masses) and the second subgroup was comprised of the remaining test isolates (except those from citrus) as well as $C$. acutatum reference isolates (corresponding to the group of isolates producing gray-olive colonies with low sporulation). On the contrary, the fast growing isolates from citrus and the reference C. gloeosporioides isolates showed 68 to $100 \%$ round-ended spores. Spore size (data not shown), however, provided very little distinction among the Colletotrichum isolates characterized in this study.

Differences in growth rate of the various Colletotrichum isolates studied were more apparent at $25^{\circ} \mathrm{C}$ (data for comparing temperatures not shown). At this temperature, all isolates could readily be differentiated into two main groups (Table 2) statistically significant at over $95 \%$. The first group was comprised of Colletotrichum isolates from Lupinus spp. and other hosts, as well as all the $C$. acutatum reference isolates, which were all slow growing (30.0 to $43.2 \mathrm{~mm}$ ). The second group included Colletotrichum isolates from citrus and the $C$. gloeosporioides reference isolates (50.2 to $70.3 \mathrm{~mm}$ ) as well as the reference isolates of $C$. falcatum, C. graminicola, and C. sublineolum (data not shown), which were all fast growing. All Colletotrichum isolates from lupin and some C. acutatum reference isolates were able to grow on PDA amend-

TABLE 2. Morphological characters (spore shape and colony diameter and color) and benomyl sensitivity of a representative set of Colletotrichum spp. isolates used in the study

\begin{tabular}{|c|c|c|c|c|c|}
\hline Isolate and host & Origin & $\begin{array}{l}\text { Spore shape } \\
\text { (\% of each class) }\end{array}$ & $\begin{array}{l}\text { Growth }(\mathrm{mm})^{\mathrm{b}} \\
5 \text { days at } 25^{\circ} \mathrm{C}\end{array}$ & Colony color ${ }^{\mathrm{c}}$ & $\begin{array}{l}\text { Mycelial growth on PDA } \\
\text { amended with benomyl }^{\text {d }}\end{array}$ \\
\hline \multicolumn{6}{|l|}{ Lupinus isolates } \\
\hline 546 & U.K. & $24-76-0$ & $43.17 \pm 1.36$ & Olive & + \\
\hline CR02 & Portugal & $0-100-0$ & $38.50 \pm 0.476$ & Gray & + \\
\hline G52 & Germany & $24-76-0$ & $37.25 \pm 1.18$ & Olive & + \\
\hline 96A4 & Australia & $16-84-0$ & $39.67 \pm 2.24$ & Olive & + \\
\hline PT30 & Azores, Portugal & $20-80-0$ & $30.00 \pm 4.76$ & Olive & + \\
\hline JR15 & Azores, Portugal & $16-80-4$ & $39.67 \pm 1.31$ & Olive & + \\
\hline HO01 & Azores, Portugal & $0-100-0$ & $34.00 \pm 0.952$ & Pink with orange masses & + \\
\hline JR03 & Azores, Portugal & $0-40-60$ & $41.00 \pm 2.46$ & Pink with orange masses & + \\
\hline \multicolumn{6}{|l|}{ Other isolates } \\
\hline CR20 & Portugal & $8-72-20$ & $41.67 \pm 1.05$ & Pink with orange masses & $\mathrm{nt}$ \\
\hline C2897 & Australia & $4-52-44$ & $39.50 \pm 1.53$ & Pink with orange masses & + \\
\hline JG05 & France & $28-68-4$ & $39.50 \pm 1.80$ & Gray & + \\
\hline CMG12 & Portugal & $24-76-0$ & $36.33 \pm 0.499$ & Olive & nt \\
\hline CR46 & Portugal & $24-76-0$ & $37.50 \pm 1.88$ & Gray & - \\
\hline TN47 & Portugal & $0-100-0$ & $32.17 \pm 1.77$ & Hyaline & + \\
\hline \multicolumn{6}{|c|}{ Reference $C$. acutatum } \\
\hline NI90-1 & U.K. & $16-68-16$ & $38.83 \pm 0.854$ & Ochre & - \\
\hline 397 & U.S.A. & $8-72-20$ & $34.67 \pm 1.19$ & Pale pink & + \\
\hline \multicolumn{6}{|c|}{ Reference C. gloeosporioides } \\
\hline 254 & Canada & $67-33-0$ & $70.33 \pm 3.44$ & Lead & - \\
\hline 315 & U.S.A. & $68-32-0$ & $67.67 \pm 4.32$ & Cream & - \\
\hline \multicolumn{6}{|l|}{ Citrus isolates } \\
\hline CR21 & Portugal & $68-32-0$ & $50.21 \pm 6.23$ & Hyaline with orange masses & - \\
\hline HO19 & Portugal & $100-0-0$ & $51.00 \pm 2.05$ & Hyaline with orange masses & - \\
\hline CR45 & Portugal & $72-28-0$ & $55.83 \pm 0.654$ & Hyaline with orange masses & - \\
\hline
\end{tabular}

$\overline{{ }^{a}}$ Spore shape values are percentage of round end spores (first value in each isolate) - one end round and the other acute (second value) - acute-ended spores (third value).

b Values are average and range of $95 \%$ confidence interval (three plates).

${ }^{\mathrm{e}}$ Descriptions based on Lardner et al. (30) and Saccardo (38).

d + denotes mycelial growth on potato dextrose agar (PDA) amended with benomyl ( $2 \mathrm{mg} \mathrm{liter}^{-1}$ ) after 5 days at $25^{\circ} \mathrm{C}$ in darkness (three replicates); - denotes no mycelial growth under these conditions; $\mathrm{nt}=$ not tested in this experiment.

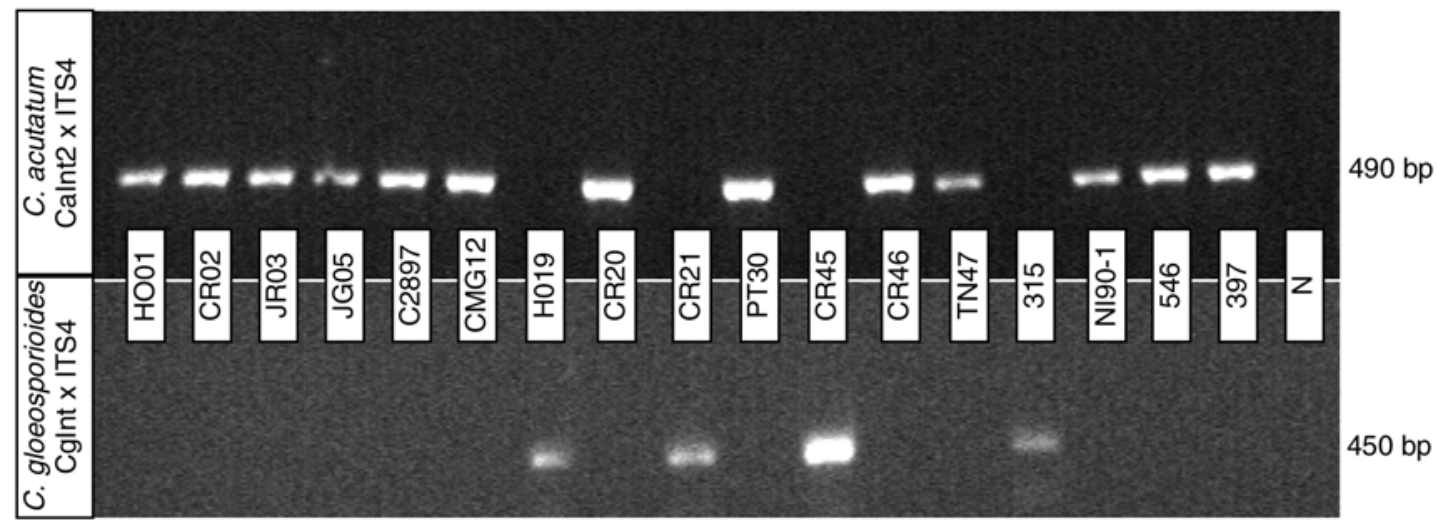

Fig. 1. Amplification products obtained in the species-specific polymerase chain reaction test (a selection of isolates is shown). All test isolates and four reference isolates (Table 1) were analyzed using primers specific for Colletotrichum acutatum and C. gloeosporioides. All test isolates (except the three isolates from citrus) gave a positive product with the $C$. acutatum-specific primer. Upper panel shows amplified products with the $C$. acutatum-specific primer for Colletotrichum isolates from lupins, five isolates from other hosts, as well as the reference C. acutatum isolates. Lower panel shows the amplified product with the $C$. gloeosporioides-specific primer for the three isolates from citrus and for reference $C$. gloeosporioides isolate. Lane $\mathrm{N}$ is the negative control. Table 1 provides isolate details. 
ed with benomyl; $C$. acutatum reference isolate NI90-1 from strawberry and Colletotrichum isolate CR46 from Vitis were the exceptions. Fast growing Colletotrichum isolates from citrus and C. gloeosporioides reference isolates were unable to grow on PDA amended with benomyl (Table 2).

Colony characteristics revealed a wide range of variation between the test and reference Colletotrichum isolates (Table 2). Isolates from Lupinus spp. could be separated into two groups. One was comprised of isolates producing pink, cottony, and low density colonies with abundant orange spore masses (isolates HO01 and JR03, but also isolates C2897 and CR20 from strawberry). The other was comprised of isolates producing gray-olive, felty and medium density colonies with little or no spore masses (corresponding to all the other isolates from Lupinus spp.). Reference $C$. acutatum isolates from other hosts present various colorations, densities, and textures, generally nearer to either pink, salmon, orange, carmine, ochre, or olive-green. Reference $C$. gloeosporioides isolates were cream, gray, or lead in colony color. The fast growing isolates from citrus and a few other test isolates did not fall into any of these categories. Table 2 summarizes the morphological characters that enabled some degree of distinction for a representative set of all test and reference isolates studied.

Diagnostic PCR with species-specific primers. In PCR tests with the $C$. acutatum-specific primer CaInt 2 used in conjunction with the conserved primer ITS4, all Colletotrichum isolates from Lupinus spp. yielded a positive 490-bp product along with the reference $C$. acutatum isolates from various other hosts (Table 1). This included isolates 96A4, 96A649, and HY09 from Lupinus spp., which were previously identified as C. gloeosporioides. Further, isolate JG05 from Ceanothus sp., previously identified as C. gloeosporioides, as well as isolates CMG12, CR20, CR46, and TN47 from different hosts, tested positive with the $C$. acutatumspecific PCR test. The C. gloeosporioides-specific primer CgInt with ITS4 yielded positive products $(450 \mathrm{bp})$ only with isolates CR21, CR45, and HO19 from citrus and the reference $C$. gloeosporioides isolates. Figure 1 shows an example of the results obtained in the species-specific PCR tests.

Sequencing of rDNA ITS region. Nucleotide sequence of the ITS1, 5.8S RNA gene, and ITS2 was determined for 49 isolates (40 from lupin and 9 from other hosts) of Colletotrichum spp. Nine reference sequences were used, mainly from either previously published data or downloaded from the EMBL database: 397 and NI90-1 (40), AF081292 and AF090853 (32), and C2897 (sequenced in this work; accession no. AJ300558) for $C$. acutatum; 315 (40) and AF090855 (32) for C. gloeosporioides; CFA12 (12) for $C$. falcatum; AF059676 for C. graminicola; and M13906 (11) for Neurospora crassa, used as the outgroup. Following multiple sequence alignment (up to 590 characters), a 502 base fully aligned region was chosen for further analyses.

Phylogenetic analysis divided the Colletotrichum isolates currently being characterized into two distinct groups designated A and B (Fig. 2; Table 1). Group A (100\% bootstrap), represented by the five reference $C$. acutatum isolates, included 40 isolates from Lupinus spp. and 5 isolates from various other hosts with $94.5 \%$ similarity. Group B (100\% bootstrap), represented by the two $C$. gloeosporioides reference isolates, included the three isolates from citrus with $99.0 \%$ similarity. Group A is divided into four subgroups designated A1, A2, A3, and A4. With $96.6 \%$ bootstrap, A1 included 38 isolates from Lupinus spp. from various countries and isolate CMG12 from cinnamon (Cinnamonium zeylanicum). All isolates in A1 showed identical sequence, except isolate HY09 from Lupinus sp., which showed a single nucleotide difference. A2 was comprised of ( $98.3 \%$ similarity) seven test isolates, including two isolates from Lupinus sp., one isolate from strawberry, and four reference $C$. acutatum isolates, three of which were from strawberry. A4 (99.6\% bootstrap) included a reference C. acutatum isolate NI90-1 and isolates JG05 and TN47 from Ceanothus sp. and Eriobotrya sp., respectively. A1 and A2 show- ed $2.8 \%$ difference, whereas A4 is further removed from these (4.1\% difference). Importantly, group A, which included the reference $C$. acutatum isolates and all the isolates from Lupinus spp., showed only $84.4 \%$ similarity to group B (essentially $C$. gloeosporioides). Both neighbor-joining and parsimony analyses, with different number of bootstrap sets, randomization options, and algorithms used, yielded comparable tree topologies, and the major groups and the subgroups observed were stable (Fig. 2). Considering all the Colletotrichum sequences aligned, 86 sites were variable (17.2\% of the alignment length), of which 57 were located in ITS1, 4 were located in 5.8S, and 25 were located in ITS2.

Sequencing of variable regions of the $t u b 2$ gene. A set of isolates, representing the various rDNA-ITS sequence groups, was chosen for determining the nucleotide sequence of two variable regions of the $t u b 2$ gene. Sequence data of the variable regions 1 and 2 were generated from 13 test isolates and reference isolates 397, C2897, and NI90-1 (C. acutatum), 315 (C. gloeosporioides),

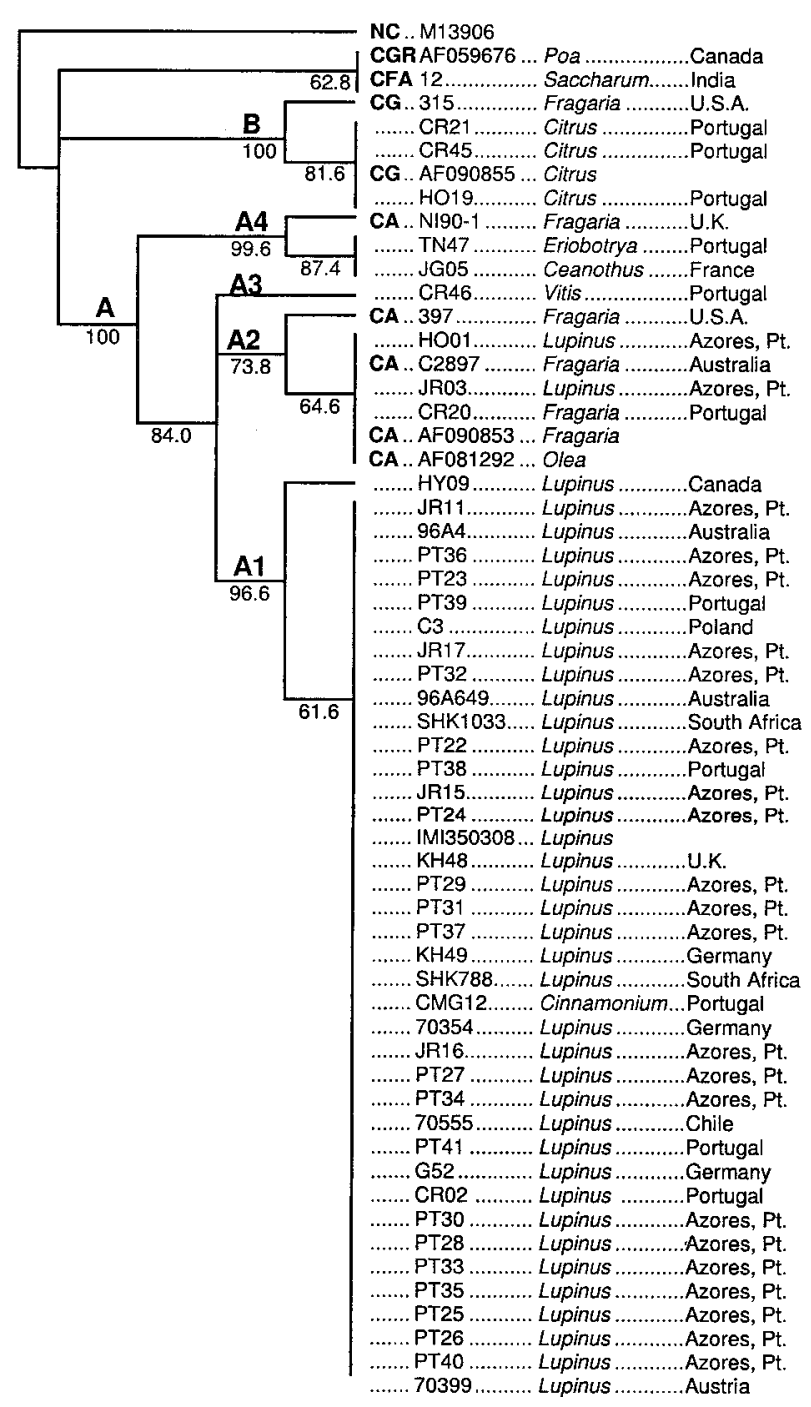

Fig. 2. Neighbor-joining consensus tree depicting relationships among Colletotrichum isolates from Lupinus spp. and other hosts, as well as C. acutatum and C. gloeosporioides isolates based on internal transcribed spacer sequences. Five hundred bootstrap data sets and Kimura-2P model distance matrices were used. Up to 10 jumbles were used for data input. Bootstrap values over $60 \%$ only are presented, other groups being collapsed to the previous node. Main groups are identified as A and B; A1 to A4 denote subgroups. Reference isolates are identified with species designations in bold (CA, C. acutatum; CFA, C. falcatum; CG, C. gloeosporioides; and CGR, C. graminicola). Neurospora crassa (NC) was used as outgroup. Database accession number is provided for reference sequences used. Pt. $=$ Portugal. Table 1 provides isolate details. 
and CFA12 (C. falcatum). Isolates U14138 (C. gloeosporioides; 10) and M34492 (C. graminicola; 36), downloaded from EMBL, were used as references and isolate M13630 (N. crassa; 35) was used as outgroup. Sequence data of the two regions for each isolate were joined to generate a global alignment $(550+488 \mathrm{bp})$ that was used for all analyses.

The test isolates were divided into two major groups, A and B (Fig. 3; Table 1), corresponding to the ITS groups. Group A ( $100 \%$ bootstrap), represented by the three reference $C$. acutatum isolates, included seven Colletotrichum isolates from Lupinus sp. and three isolates from other hosts. Group B (100\% bootstrap), represented by the two reference C. gloeosporioides isolates, included the three fast growing isolates from citrus with $97.0 \%$ similarity. Group A could be divided into four subgroups (A1, A2, A3, and A4) as with ITS data. A1 (100\% bootstrap) was comprised of five isolates from Lupinus with identical sequence. A2 (100\% bootstrap) included two isolates from Lupinus sp. and three from strawberry, two of which were reference $C$. acutatum isolates 397 and C2897. Group A had an overall similarity of $91.4 \%$ and showed only $80.2 \%$ similarity to group B. Within group A, A1 showed $97.7 \%$ similarity to A2, while A1 and A2 were $95.2 \%$ similar to A4. C. falcatum and C. graminicola clustered and fit between group A and B (81.2\% bootstrap) (Fig. 3). Overall, tree topology was consistent (data not shown) with both parsimony and neighbor-joining analysis. Analyzing the variable regions 1 and 2 separately also yielded comparable topologies, particularly with respect to the clear separation of the major groups $\mathrm{A}$ and $\mathrm{B}$. However, with region 1 alone, A2 and A4 clustered together, and C. graminicola and C. falcatum cluster was outside of the A and B groups cluster (data not shown).

Considering all the Colletotrichum sequences aligned, 285 sites were variable ( $27.1 \%$ of alignment length), 173 were located in region 1 , and 92 were located in region 2 .

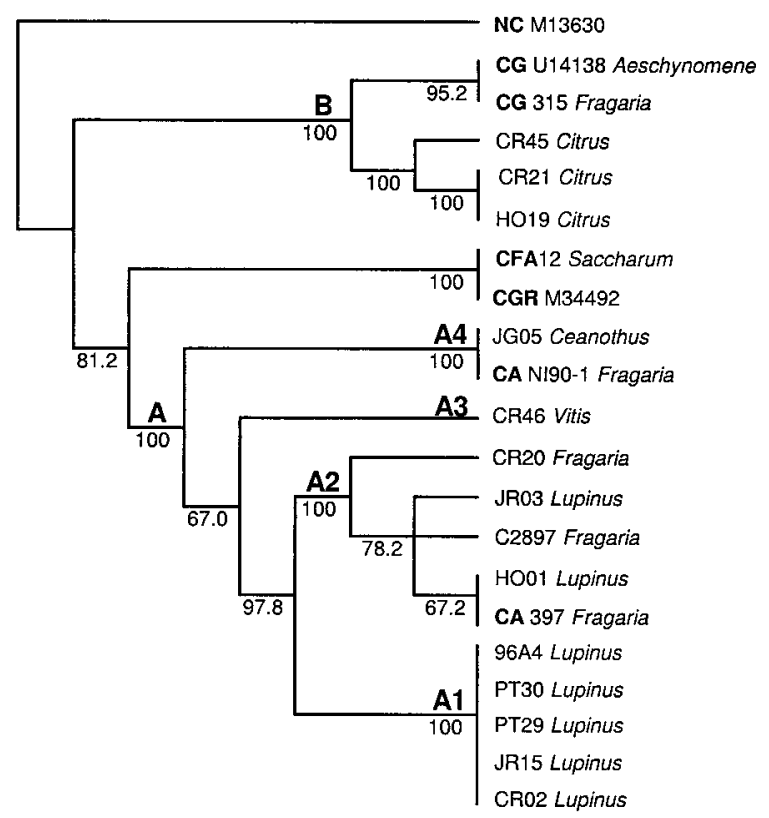

Fig. 3. Neighbor-joining consensus tree depicting relationships among $\mathrm{Col}$ letotrichum isolates from Lupinus spp. and other hosts, as well as reference C. acutatum and C. gloeosporioides isolates based on $\beta$-tubulin 2 (tub2) gene sequences. Five hundred bootstrap data sets and Kimura-2P model distance matrices were used. Up to 10 jumbles were used for data input. Bootstrap values over $60 \%$ only are presented, other groups being collapsed to the previous node. Main groups are identified as A and B; A1 to A4 relate to subgroups observed with internal transcribed spacer data. Reference isolates are identified with species designations in bold (CA, C. acutatum; CFA, $C$. falcatum; CG, C. gloeosporioides; and CGR, C. graminicola). Neurospora crassa (NC) was used as outgroup. Database accession number is provided for reference sequences used. Table 1 provides isolate details.
Sequencing of a variable region in his 4 gene. Nucleotide sequence of a variable region (257 bp) in the his4 gene was generated for a representative set of eight test isolates and six reference isolates: 318, 397, 546, and NI90-1 (C. acutatum); 315 (C. gloeosporioides); and 84032 (C. graminicola). N. crassa sequence (X01611) (51) accessed from EMBL was used as outgroup.

The test isolates were divided into two major groups, A and B (Fig. 4; Table 1), corresponding to the ITS and tub2 sequence groups. Group A ( $92.0 \%$ bootstrap), represented by the four reference $C$. acutatum isolates, included Colletotrichum isolates from Lupinus sp. as well as other hosts, with $84.8 \%$ similarity. Group B (99.4\% bootstrap), represented by the reference $C$. gloeosporioides, included the two fast growing isolates from citrus, with $94.6 \%$ similarity. The similarity between groups A and B was only $77.8 \%$.

Some of the subgroups observed within group A (Fig. 4) were comparable to ITS and tub2 results. For example, subgroup A4 with isolates JG05 and reference $C$. acutatum NI90-1 was maintained with $99.2 \%$ similarity, as well as subgroup A1 with isolates 546, CR02, and PT30 (99.6\% similarity). Isolate CR46 clustered with reference $C$. acutatum 318 with $97.7 \%$ similarity (subgroup A3). Subgroup A2 isolates HO01 and JR03 clustered with 95.2\% bootstrap, but not the $C$. acutatum reference isolate 397 (Fig. 4). Overall, tree topology was consistent (data not shown) with both parsimony and neighbor-joining analysis. Considering all Colletotrichum sequences aligned, 49 informative sites (19.1\% of the sequenced length) were found.

AP-PCR analysis. AP-PCR analysis was performed with four arbitrary primers to characterize 67 Colletotrichum isolates in all. This included 49 test isolates (41 of these were from Lupinus spp.) and 18 reference isolates (12 C. acutatum, 2 C. falcatum, $2 C$. gloeosporioides, 1 C. graminicola, and $1 C$. sublineolum). On an average, each primer amplified 8 to 9 bands per isolate and 31 to 32 characters were scored per primer with all isolates, none being monomorphic (Fig. 5).

Cluster analysis revealed several groups (Fig. 6; Table 1), some of which corresponded to those recorded with ITS, tub2 and his 4 sequences, and morphological characters. For example, 39 Colletotrichum isolates from Lupinus spp. from various countries

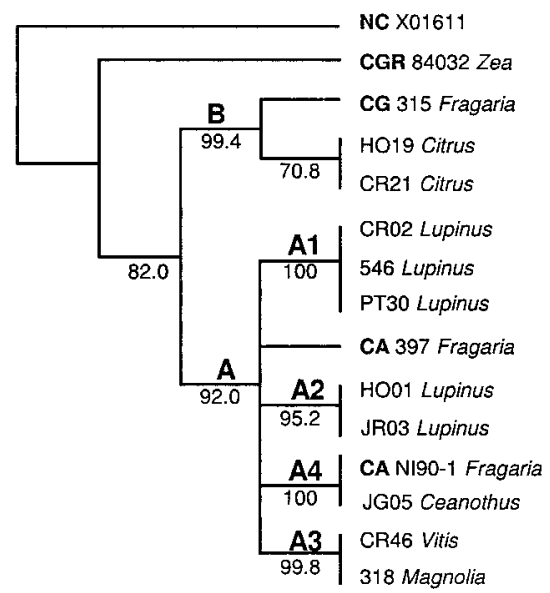

Fig. 4. Neighbor-joining consensus tree depicting relationships among Colletotrichum isolates from Lupinus spp. and other hosts, as well as reference C. acutatum and C. gloeosporioides isolates based on histone 4 gene sequences. Five hundred bootstrap data sets and Kimura-ZP model distance matrices were used. Up to 10 jumbles were used for data input. Bootstrap values over $60 \%$ only are presented, other groups being collapsed to the previous node. Main groups are identified as A and B; A1 to A4 relate to subgroups observed with internal transcribed spacer data. Reference isolates are identified with species designations in bold (CA, C. acutatum; CG, C. gloeosporioides; and CGR, C. graminicola). Neurospora crassa (NC) was used as outgroup. Table 1 provides isolate details. 
formed a homogeneous group along with a single isolate from cinnamon with $79.6 \%$ bootstrap, corresponding to subgroup A1 observed in the other analyses. Subgroup A2 isolates HO01 and JR03 (from Lupinus sp.) clustered with reference isolate C2897 from strawberry (98.8\% bootstrap). Other groups that were also observed previously included (Fig. 6) isolates JG05 and TN47, and reference $C$. acutatum isolate NI90-1 (98.8\% bootstrap; subgroup A4), and three fast growing isolates (CR21, CR45, and HO19) from citrus (73.6\% bootstrap; group B). However, the relationships between these relatively well-supported groups were far from clear, because the bootstrap values were low for the basal branches or nodes connecting these groups. This was observed independent of the parsimony or neighbor-joining analyses and the different tested parameters. Consequently, the relationship between the test isolates and reference species such as $C$. acutatum and $C$. gloeosporioides was not as clear-cut as with the ITS, $t u b 2$, and his 4 sequence results. The AP-PCR analysis was more useful in assessing the intraspecific diversity rather than in assigning the test isolates to a particular species.

AFLP analysis. Thirty-three Colletotrichum isolates, representing the entire range of diversity observed with other markers or characters, were analyzed with four primer combinations. This included 15 test isolates and 18 reference isolates $(12$ C. acutatum, 2 C. falcatum, 2 C. gloeosporioides, 1 C. graminicola, and $1 C$. sublineolum). On average, primer combinations with three selective nucleotides yielded 86 bands per isolate and primer pair, whereas primer combinations with four selective nucleotides yielded only 41 bands per isolate and primer pair. The AFLP analysis yielded an average of 376 characters for all isolates per primer combination, and none were monomorphic.

Colletotrichum isolates from Lupinus spp. fell into two subgroups, both supported by $100 \%$ bootstrap values (Fig. 7). These corresponded to subgroups A1 and A2 from previous analyses (Fig. 7). The fast growing isolates CR21, CR45, and HO19 from citrus clustered with the two reference $C$. gloeosporioides isolates (80.8\% bootstrap) and corresponded to the group B observed earlier. Similar to AP-PCR, test isolate CR46 from Vitis sp. clustered with various $C$. acutatum reference isolates (100\% bootstrap; subgroup A3); isolates JG05 and TN47 and the reference $C$. acutatum isolate NI90-1 formed a subgroup (A4) with $100 \%$ bootstrap (Fig. 7). As observed with AP-PCR analysis, the bootstrap values were low for basal branches/nodes connecting these groups, independent of the method of analysis.

\section{DISCUSSION}

The objectives of the present study were to understand the genetic and morphological diversity of the lupin anthracnose pathogen and its relationship to Colletotrichum spp. causing anthracnose on other hosts, and to use this knowledge to ascertain the pathogen species designation, which remains unresolved as either C. gloeosporioides $(13,52)$, C. acutatum $(24,30,41)$, or a new species (27).

The genetic diversity among Colletotrichum isolates causing lupin anthracnose was very low with all the molecular markers analyzed, as they were divided into only two distinct subgroups A1 and A2, corresponding to the broad groups observed with spore shape and colony color. Subgroup A1 included 38 isolates representing worldwide locations. Subgroup A1, despite the diverse origin of the isolates, appears to be genetically homogeneous and corresponds to lupin anthracnose pathogen groups COL2 (52) and D (30), previously identified based on VCG and RAPDs. A Canadian lupin anthracnose isolate was previously categorized as COL1 (52). However, isolate HY09, originating from Canada, showed very high similarity in AP-PCR and AFLP analyses and only a single base difference in ITS sequence to other isolates in subgroup A1 (corresponding to COL2 referred above). This is not entirely surprising, because COL1, hypothesized as causing the early lupin anthracnose outbreaks in the United States and spread to Canada and Europe (39), was not found in a recent survey in the central and eastern United States (13). Moreover, lupin anthracnose isolates HO01 and JR03, from the Azores islands (in the mid-north Atlantic Ocean), Portugal, formed subgroup A2 and were clearly separated from the homogeneous subgroup A1. No more isolates belonging to A2 were recorded from Azores despite further surveys. L. angustifolius wild germ plasm accessions resistant to early lupin anthracnose outbreaks were found (14) and a resistance gene $A n$ was identified (49). However, cultivars bearing $A n$ are susceptible to the contemporary pathogen subgroups A1 and A2 (P. Talhinhas, J. NevesMartins, and $\mathrm{H}$. Oliveira, unpublished data).

Isolates HO01 and JR03 (A2) from lupin consistently clustered with test (CR20) and reference (397 and C2897) C. acutatum isolates from strawberry with all the markers. Based on isolate 397 (strawberry, United States), A2 corresponds to previously identified $C$. acutatum groups pathogenic to strawberries such as CA3, COL3, and C1-SC3 $(17,20,40,52)$. In view of the homogeneity of the A1 isolates from lupin and the close relationship of A2 isolates

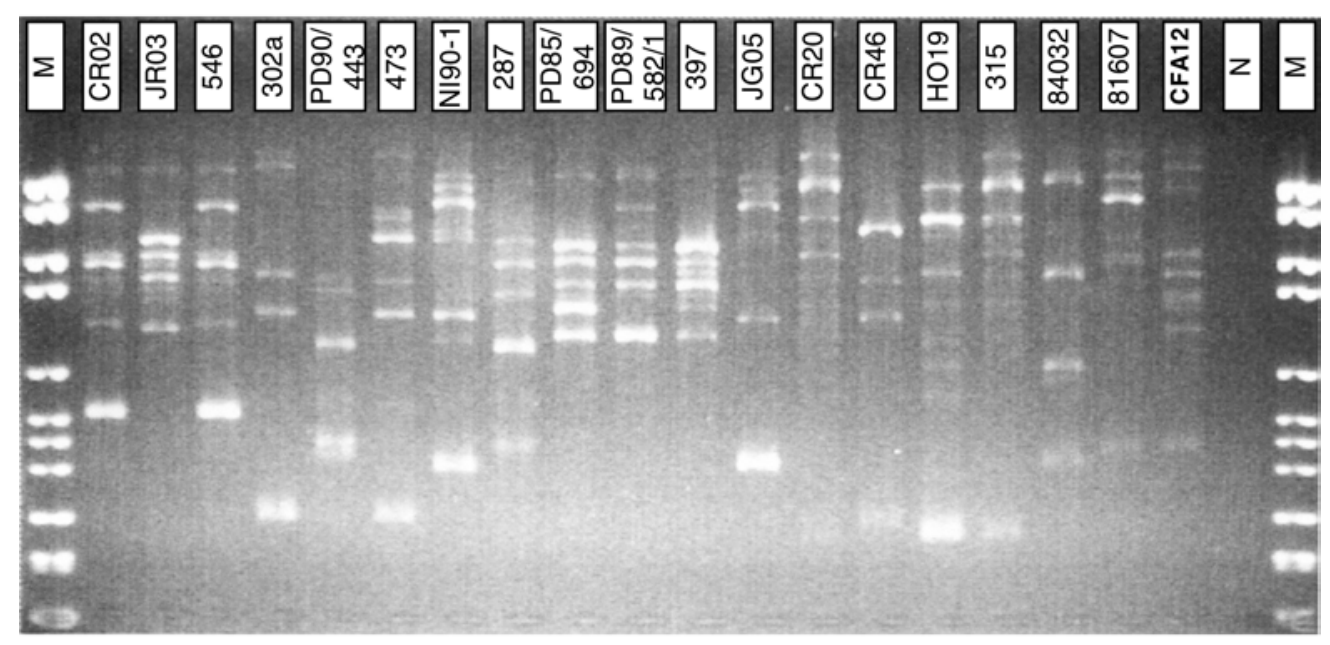

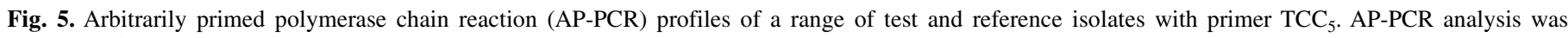

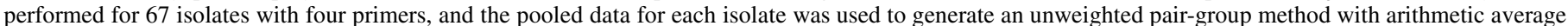

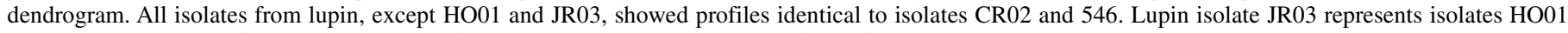

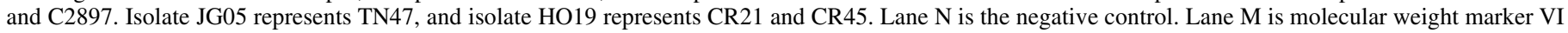
(Roche Diagnostics, Mannheim, Germany). Table 1 provides isolate details. 
from lupin to $C$. acutatum groups from strawberry, it could be speculated that A2 might have originated from strawberry pathogens, but this needs to be further investigated. It is interesting that C. acutatum VCG ANE-F infecting anemone (Anemone coronaria) was also considered to be from strawberry (19). It has been hypothesized (30) that episodic selection (4) could have occurred within asexual populations of $C$. acutatum sensu lato in New Zealand, giving rise to new host-specific forms. On the other hand, recent report of Glomerella acutata, the teleomorph of $C$. acutatum, suggests that dissemination of potentially sexually recombining strains and evolution and adaptation to new and diverse hosts is a possibility (25). Further, subgroup A4 observed in this study also comprised Colletotrichum isolates from Ceanothus sp. and Eriobotrya sp. (Table 1) and reference C. acutatum isolate NI90-1 from strawberry. The subgroup A4 appears to be related to the European strawberry $C$. acutatum groups CA1 (40), ACUT Ia (8), and C1-SC5 (20). Among the Colletotrichum isolates tested from a few other hosts, CR46 from Vitis sp., representing sub-

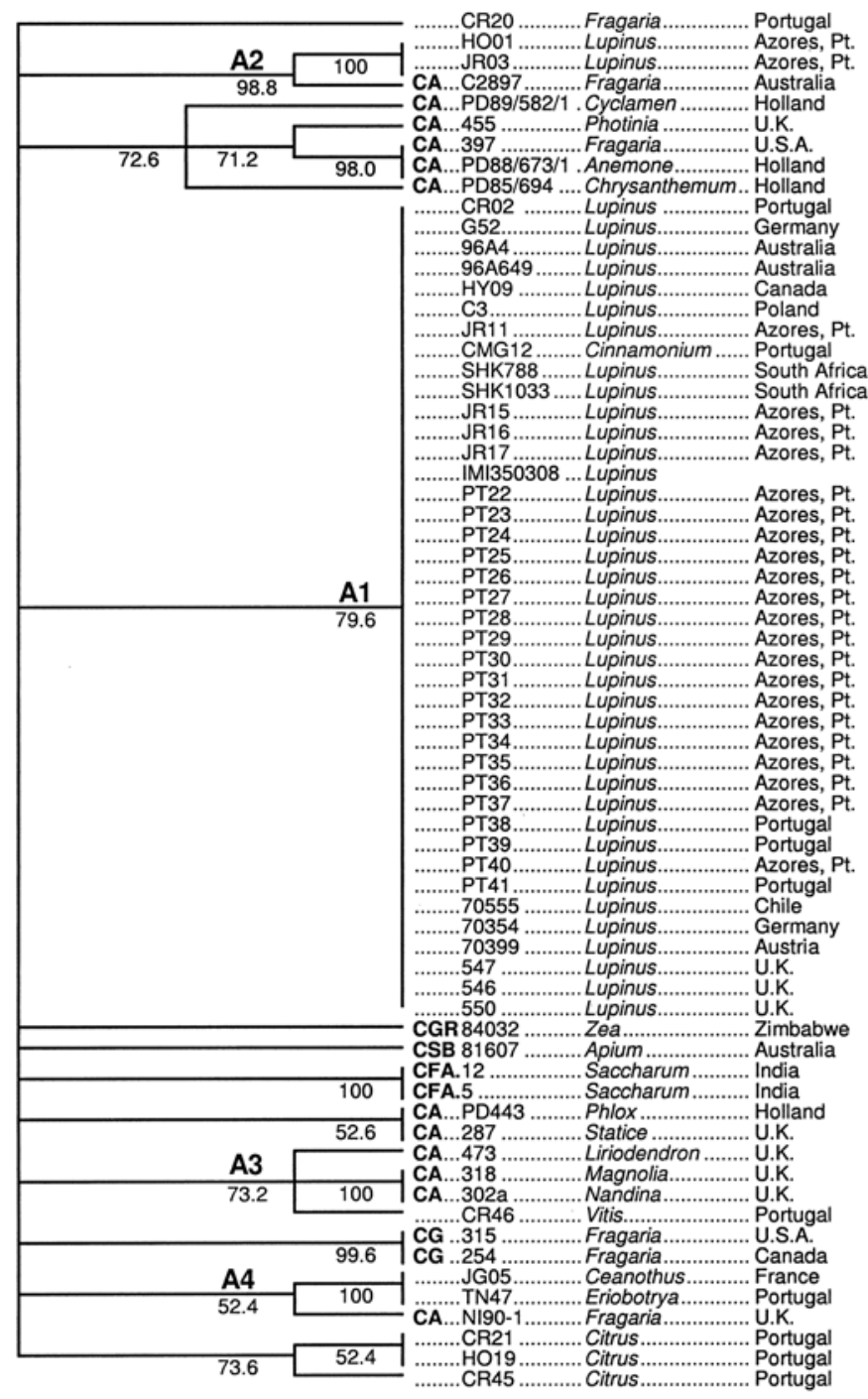

Fig. 6. Unweighted pair-group method with arithmetic average dendrogram showing diversity and relationships among Colletotrichum isolates from Lupinus spp. and other hosts, as well as reference C. acutatum and C. gloeosporioides isolates based on arbitrarily primed polymerase chain reaction analysis. Five hundred bootstrap data sets and Dice distance matrices were used. Bootstrap values over $50 \%$ only are presented, other groups being collapsed to the previous node. A1 to A4 relate to subgroups observed with internal transcribed spacer data. Reference isolates are identified with species designations in bold (CA, C. acutatum; CFA, C. falcatum; CG, $C$. gloeosporioides; CGR, C. graminicola; and CSB, C. sublineolum $)$. Pt. = Portugal. Table 1 provides isolate details. group A3, clustered with $C$. acutatum reference isolates from several ornamentals including Magnolia sp. Isolate CMG12 from cinnamon formed part of subgroup A1, which only contained the relatively homogenous Colletotrichum isolates from lupin. The host range and epidemiology of various genetic groups within $C$. acutatum clearly needs to be further investigated.

Both AP-PCR and AFLP analyses were very useful in assessing the intraspecific diversity of Colletotrichum spp., and a number of groups corresponded to those observed with the ITS, tub2, and his 4 analyses. The basal branches linking the AP-PCR/AFLP groups had low bootstrap values. Although AFLP analysis offered relatively better resolution in relating the groups, neither were useful at the interspecific level, presumably because these markers reveal more genetic distance between the groups $(8,17)$. It is important to recognize this and use an integrated multilevel approach using gene sequences as well as genome-wide markers to accurately assess the intra- and interspecific relationships in fungal populations and taxonomic groups.

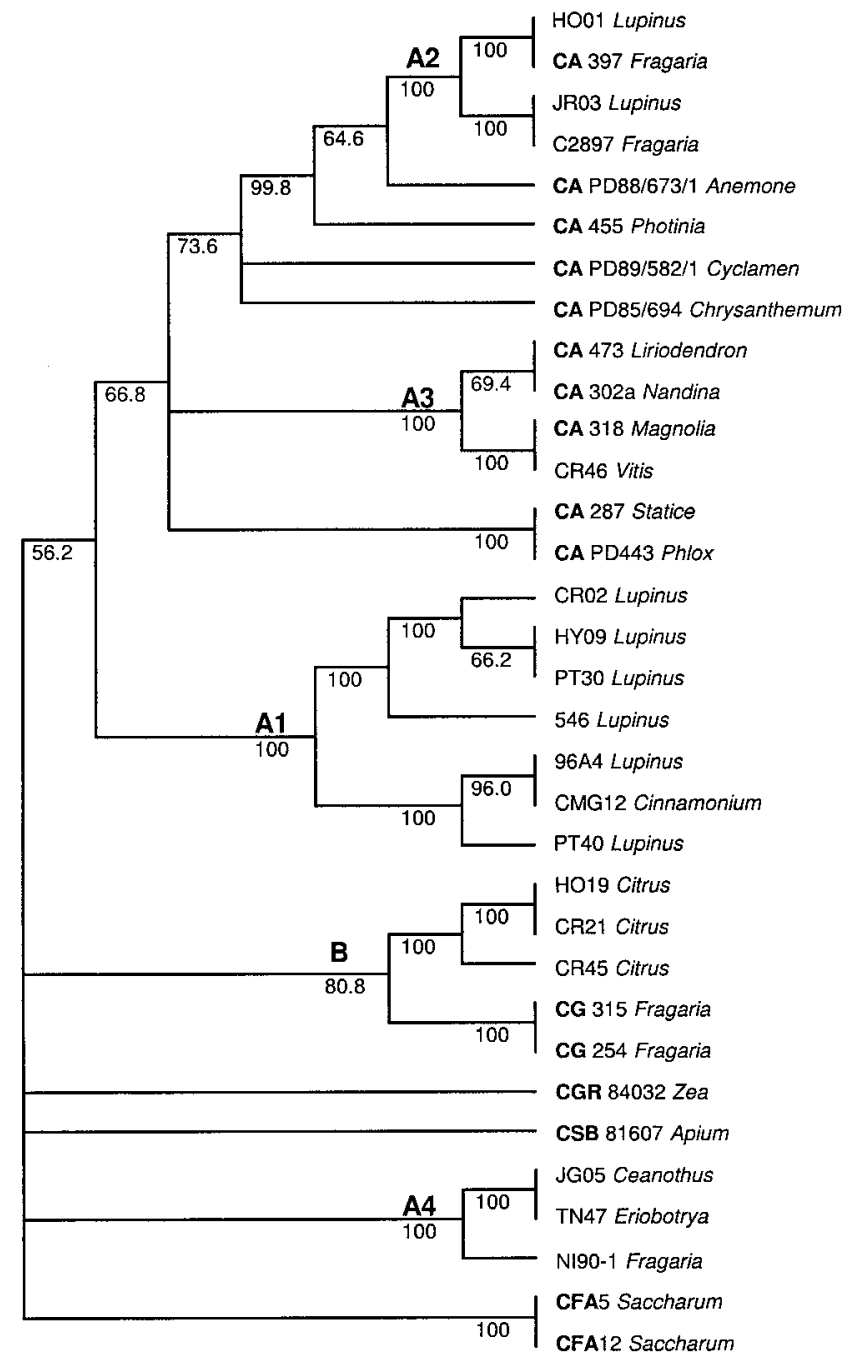

Fig. 7. Unweighted pair-group method with arithmetic average dendrogram showing diversity and relationships among Colletotrichum isolates from Lupinus spp. and other hosts, as well as reference C. acutatum and C. gloeosporioides isolates based on amplified fragment length polymorphism analysis. Five hundred bootstrap data sets and Dice distance matrices were used. Bootstrap values over $50 \%$ only are presented, other groups being collapsed to the previous node. A1 to A4 relate to subgroups observed with internal transcribed spacer data. Reference isolates are identified with species designations in bold (CA, C. acutatum; CFA, C. falcatum; CG, C. gloeosporioides; CGR, C. graminicola; and CSB, C. sublineolum). Table 1 provides isolate details. 
The species diagnostic value of most of the morphological characters studied appears to be limited. Spore shape and colony growth rate were the exceptions and placed the lupin anthracnose pathogen closer to $C$. acutatum than $C$. gloeosporioides, which is in agreement with species-specific PCR, ITS, tub2, and his4 sequence analyses. All Colletotrichum isolates from Lupinus spp. tested positive with the $C$. acutatum-specific primer along with the reference $C$. acutatum isolates, strongly suggesting that the lupin anthracnose pathogen is $C$. acutatum. These isolates showed a higher proportion of spores with one round end and one acute-end (40 to $100 \%$ ) and spores acute at both ends (0 to 60\%) than spores with round ends (0 to $28 \%$ ) and were slower growing than $C$. gloeosporioides. Isolates from citrus and the $C$. gloeosporioides references were the only ones to react positively with the $C$. gloeosporioides-specific primer and showed 68 to $100 \%$ roundended spores and 0 to $32 \%$ spores with one round end and one acute end, and no spores acute at both ends. Differential sensitivity to benomyl has been used to distinguish $C$. acutatum and $C$. gloeosporioides (1) and present results are in general agreement with this, except isolates NI90-1 and CR46, which consistently grouped with $C$. acutatum in all molecular analyses, but were sensitive to benomyl. C. acutatum- and C. gloeosporioides-specific primers have been used with a number of hosts to distinguish these species $(1,7,15,17)$ because of the difficulties encountered in the sole use of morphological characters $(26,28,30,32,44)$. Present results are contrary to reports by Yang and Sweetingham (52) and Elmer et al. (13), who described the lupin anthracnose pathogen as C. gloeosporioides, although their isolates had considerable proportion of clavate conidia and low similarity in RAPDs to $C$. gloeosporioides. They also suggested that the cultural pigmentation and colony margin were useful characters (52). However, variation in colony color/pigmentation within single-spore cultures and between sectors within a single-spore culture has been observed in Colletotrichum spp. (26,28,31). Moreover, recognition of several morphological groups within C. acutatum sensu lato isolates from a wide range of hosts including lupins and variability in the shape of the conidia $(26,30)$ as well as the influence of the growth medium on the shape and size of the conidia in $C$. acutatum $(1,15)$ further support the present results.

Extensive analysis of the ITS sequence data from 49 test isolates and well established $C$. acutatum and $C$. gloeosporioides references grouped the 40 isolates from Lupinus spp. with $C$. acutatum (group A, $100 \%$ bootstrap and $94.5 \%$ similarity) and the fast growing isolates from citrus with $C$. gloeosporioides (group B, $100 \%$ bootstrap and $99.0 \%$ similarity). Similarity between groups A and B, representing C. acutatum and C. gloeosporioides, respectively, was only $84.4 \%$. Thus, the lupin pathogen isolates, identified as $C$. acutatum in the PCR test, showed more than $15.0 \%$ difference to $C$. gloeosporioides and only 0 to $5.5 \%$ difference to $C$. acutatum, comparable to $5.6 \%$ difference in ITS previously reported within isolates of this species pathogenic to strawberry (40). In the present study, tub2 and his4 sequence analyses also grouped the isolates from Lupinus spp. with $C$. acutatum (group A, 92.0 to $100 \%$ bootstrap) and the isolates from citrus with C. gloeosporioides (group B, 99.4 to $100 \%$ bootstrap) concordant with ITS results.

To our knowledge, this is the first use of tub2 and his4 gene sequences to assess intra- and interspecific as well as taxonomic relationships in the genus Colletotrichum, and the results obtained clearly show their potential. Moreover, use of tub2 and his 4 gene sequences to verify the phylogenetic groupings obtained with ITS helps to overcome the potential limitation of divergent ITS copies existing within single-spore isolates (29).

In the last few years, existence of intermediate isolates showing a continuum of morphological and colony characters between $C$. acutatum and C. gloeosporioides has been confirmed (16) and, based on molecular characters, the anthracnose pathogen previously described as $C$. gloeosporioides was redesignated as $C$. acutatum on a number of hosts $(15,17,19,28,32)$. Present results clearly substantiate previous reports based on limited ITS (41), rDNA-restriction fragment length polymorphism (45), and RAPD (30) data that the lupin anthracnose pathogen from worldwide sources is $C$. acutatum, underlining the utility of species-specific PCR for pathogen diagnosis. Lupin anthracnose pathogen mainly represented by $\mathrm{A} 1$ is homogenous, except the few isolates belonging to A2. Based on this knowledge, we believe that this pathogen should be designated as $C$. acutatum and a subspecific taxon could be created to alleviate the concerns (26) of an excessive enlargement of the species.

Genetic homogeneity of A1 also suggests a certain degree of host specificity in this pathogen that needs to be investigated. Present results also underline the necessity for not only strict quarantine measures on the movement of lupin seed to contain further spread of the pathogen, but more importantly, the identification and development of resistance in lupins, particularly to A1, which is present across the globe.

\section{ACKNOWLEDGMENTS}

This research was supported in part by grants PRAXIS/P/AGR/ 11201/98 and PRAXIS/BD/11469/97 (award to P. Talhinhas) from Fundação para a Ciência e a Tecnologia (Portugal). Molecular characterization was carried out at HRI under license PHL 5G/3683 (3/2001). We thank A. Brown, C. Rego, J. Rocha, H. Yang, H. Nirenberg, S. Koch, J. Gondran, K. Hughes, I. Frencel, C. Moura-Guedes, and T. Nascimento for providing biological material, and R. Edwards and L. Grenville for help with sequencing and AFLP work.

\section{LITERATURE CITED}

1. Adaskaveg, J. E., and Hartin, R. J. 1997. Characterization of Colletotrichum acutatum isolates causing anthracnose of almond and peach in California. Phytopathology 87:979-987.

2. Agostini, J. P., Timmer, L. W., and Mitchell, D. J. 1992. Morphological and pathological characteristics of strains of Colletotrichum gloeosporioides from citrus. Phytopathology 82:1377-1382.

3. Bernstein, B., Zehr, E. I., Dean, R. A., and Shabi, E. 1995. Characteristics of Colletotrichum from peach, apple, pecan, and other hosts. Plant Dis. 79:478-482.

4. Brasier, C. M. 1995. Episodic selection as a force in fungal microevolution, with special reference to clonal speciation and hybrid introgression. Can. J. Bot. 73:S1213-S1221.

5. Bridge, P. D., Pearce, A., Rivera, A., and Rotherford, M. A. 1997. VNTR derived oligonucleotides as PCR primers for population studies in filamentous fungi. Lett. Appl. Microbiol. 24:426-430.

6. Brown, A., and Soepena, H. 1994. Pathogenicity of Colletotrichum acutatum and C. gloeosporioides on leaves of Hevea spp. Mycol. Res. 98:264-266.

7. Brown, A. E., Sreenivasaprasad, S., and Timmer, L. W. 1996. Molecular characterization of slow-growing orange and key lime anthracnose strains of Colletotrichum from citrus as C. acutatum. Phytopathology 86:523-527.

8. Buddie, A., Martínez-Culebras, P., Bridge, P., García, M., Querol, A., Cannon, P., and Monte, E. 1999. Molecular characterization of Colletotrichum strains derived from strawberry. Mycol. Res. 103:385394.

9. Buhr, T. L., and Dickman, M. B. 1993. Isolation and characterization of a beta-tubulin gene from Colletotrichum gloeosporioides f. sp. aeschynomene. Gene 124:121-125.

10. Buhr, T. L., and Dickman, M. B. 1994. Isolation, characterization, and expression of a second beta-tubulin-encoding gene from Colletotrichum gloeosporioides f. sp. aeschynomene. Appl. Environ. Microbiol. 60:4155-4159.

11. Chambers, C., Dutta, S. K., and Crouch, R. J. 1986. Neurospora crassa ribosomal DNA: Sequence of internal transcribed spacer and comparison with $N$. intermedia and $N$. sitophila. Gene 44:159-164.

12. Chipili, J. 1996. The genetic diversity in Colletotrichum falcatum and its relationship to $C$. graminicola and $C$. sublineolum. M.Phil. thesis. The Queen's University of Belfast, United Kingdom.

13. Elmer, W., Yang, H., and Sweetingham, M. 2001. Characterization of Colletotrichum gloeosporioides isolates from ornamental lupines in Connecticut. Plant Dis. 85:216-219.

14. Forbes, I., and Wells, H. D. 1961. Inheritance of resistance to 
anthracnose in blue lupines, Lupinus angustifolius L. Crop Sci. 1:139141.

15. Förster, H., and Adaskaveg, J. 1999. Identification of subpopulations of Colletotrichum acutatum and epidemiology of almond anthracnose in California. Phytopathology 89:1056-1065.

16. Freeman, S., Katan, T., and Shabi, E. 1998. Characterization of Colletotrichum species responsible for anthracnose diseases of various fruits. Plant Dis. 82:596-605.

17. Freeman, S., Minz, D., Jurkevitch, E., Maymon, M., and Shabi, E. 2000. Molecular analyses of Colletotrichum species from almond and other fruits. Phytopathology 90:608-614.

18. Freeman, S., Pham, M., and Rodriguez, R. 1993. Molecular genotyping of Colletotrichum species based on arbitrarily primed PCR, A+T-rich DNA, and nuclear DNA analyses. Exp. Mycol. 17:309-322.

19. Freeman, S., Shabi, E., and Katan, T. 2000. Characterization of Colletotrichum acutatum causing anthracnose of anemone (Anemone coronaria L.). Appl. Environ. Microbiol. 66:5267-5272.

20. García-Muñoz, J., Suárez, M., Grondona, I., Monte, E., Buddie, A., Bridge, P., and Cannon, P. 2000. A physiological and biochemical approach to the systematics of Colletotrichum species pathogenic to strawberry. Mycologia 92:488-498.

21. Gilbert, J. E., Lewis, R. V., Wilkinson, M. J., and Caligari, P. D. S. 1999. Developing an appropriate strategy to assess genetic variability in plant germplasm collections. Theor. Appl. Genet. 98:1125-1131.

22. Glass, N. L., and Donaldson, G. C. 1995. Development of primer sets designed for use with the PCR to amplify conserved genes from filamentous ascomycetes. Appl. Environ. Microbiol. 61:1323-1330.

23. Gondran, J. 1991. The diseases of white lupin crops in France-Prevention possibilities. Pages 277-279 in: Proc. Int. Lupin Conf., VIth. D. von Baer, ed. Asociación Chilena del Lupino, Temuco, Chile.

24. Gondran, J., and Pacault, D. 1997. L'anthracnose du lupin blanc. Phytoma 494:28-31.

25. Guerber, J. C., and Correll, J. C. 2001. Characterization of Glomerella acutata, the teleomorph of Colletotrichum acutatum. Mycologia 93:216229.

26. Johnston, P., and Jones, D. 1997. Relationships among Colletotrichum isolates from fruit-rots assessed using rDNA sequences. Mycologia 89:420-430.

27. Koch, S. 1996. Colletotrichum spp. on dry beans and lupins in South Africa. Ph.D. thesis. University of Pretoria, South Africa.

28. Kuramae-Izioka, E. E., Lopes, C. R., Souza, N. L., and Machado, M. A. 1997. Morphological and molecular characterization of Colletotrichum spp. from citrus orchards affected by postbloom fruit drop in Brazil. Eur. J. Plant Pathol. 103:323-329.

29. Lanfranco, L., Delpero, M., and Bonfante, P. 1999. Intrasporal variability of ribosomal sequences in the endomycorrhizal fungus Gigaspora margarita. Mol. Ecol. 8:37-45.

30. Lardner, R., Johnston, P., Plummer, K., and Pearson, M. 1999. Morphological and molecular analysis of Colletotrichum acutatum sensu lato. Mycol. Res. 103:275-285.

31. Liyanage, H. D., McMillan, R. T., and Kistler, H. C. 1992. Two genetically distinct populations of Colletotrichum gloeosporioides from citrus. Phytopathology 82:1371-1376.

32. Martín, M., and García-Figueres, F. 1999. Colletotrichum acutatum and C. gloeosporioides cause anthracnose on olives. Eur. J. Plant Pathol. 105:733-741.

33. Mills, P. R., Sreenivasaprasad, S., and Brown, A. E. 1992. Detection and differentiation of Colletotrichum gloeosporioides isolates using PCR. FEMS Microbiol. Lett. 98:137-144.

34. Muthumeenakshi, S. 1996. Molecular taxonomy of the genus Trichoder$m a$. Ph.D. thesis. The Queen's University of Belfast, United Kingdom.
35. Orbach, M. J., Porro, E. B., and Yanofsky, C. 1986. Cloning and characterization of the gene for beta-tubulin from a benomyl-resistant mutant of Neurospora crassa and its use as a dominant selectable marker. Mol. Cell Biol. 6:2452-2461.

36. Panaccione, D. G., and Hanau, R. M. 1990. Characterization of two divergent $\beta$-tubulin genes from Colletotrichum graminicola. Gene 86:163170 .

37. Rodriguez, R., and Yoder, O. 1991. A family of conserved repetitive DNA elements from the fungal plant pathogen Glomerella cingulata (Colletotrichum lindemuthianum). Exp. Mycol. 15:232-242.

38. Saccardo, P. A. 1891. Chromotaxia seu nomenclator colorum polyglottus adclitis speciminibus coloratis ad botanicorum et zoologorum. Patavii, Italiae.

39. Shivas, R. G., McClements, J. L., and Sweetingham, M. W. 1998. Vegetative compatibility amongst isolates of Colletotrichum causing lupin anthracnose. Austral. Plant Pathol. 27:269-273.

40. Sreenivasaprasad, S., Brown, A. E., and Mills, P. R. 1992. DNA sequence and interrelationships among Colletotrichum species causing strawberry anthracnose. Physiol. Mol. Plant Pathol. 41:265-281.

41. Sreenivasaprasad, S., Mills, P. R., and Brown, A. E. 1994. Nucleotide sequence of the rDNA spacer 1 enables identification of isolates of Colletotrichum as C. acutatum. Mycol. Res. 98:186-188.

42. Sreenivasaprasad, S., Mills, P. R., Meehan, B. M., and Brown, A. E. 1996. Phylogeny and systematics of 18 Colletotrichum species based on ribosomal DNA spacer sequences. Genome 39:499-512.

43. Sreenivasaprasad, S., Sharada, K., Brown, A. E., and Mills, P. R. 1996. PCR-based detection of Colletotrichum acutatum on strawberry. Plant Pathol. 45:650-655.

44. Sutton, B. C. 1992. The genus Glomerella and its anamorph Colletotrichum. Pages 1-26 in: Colletotrichum: Biology, Pathology and Control. J. A. Bailey and M. J. Jeger, eds. CAB International, Wallingford, UK.

45. Talhinhas, P., Martins, J. N., Oliveira, H., and Sreenivasaprasad, S. 1998. Variabilidade genética numa espécie de Colletotrichum causadora de antracnose do tremoceiro e avaliação de resistência em germoplasma de Lupinus spp. Pages 303-310 in: Actas Reunião Bienal Soc. Portug. Fitopatol. J. Sequeira, M. Borges, and M. Jorge-Silva, eds. Soc. Portug. Fitopatol., Oeiras, Portugal.

46. Thompson, J. D., Higgins, D. G., and Gibson, T. J. 1994. CLUSTAL W: Improving the sensitivity of progressive multiple sequence alignment through sequence weighting, positions-specific gap penalties and weight matrix choice. Nucleic Acids Res. 22:4673-4680.

47. Vos, P., Hogers, R., Bleeker, M., Reijans, M., Vandelee, T., Hornes, M., Frijters, A., Pot, J., Peleman, J., Kuiper, M., and Zabeau, M. 1995. AFLP-A new technique for DNA-fingerprinting. Nucleic Acids Res. 23:4407-4414.

48. Weimer, J. L. 1943. Anthracnose of lupines. Phytopathology 33:249-252.

49. Wells, H. D., and Forbes, I. 1967. Effects of temperature on growth of Glomerella cingulata in vitro and its pathogenicity to Lupinus angustifolius genotypes an an and An An. Phytopathology 57:1309-1311.

50. White, T. J., Bruns, T., Lee, S., and Taylor, J. 1990. Amplification and direct sequencing of fungal ribosomal RNA genes for phylogenetics. Pages 315-322 in: PCR Protocols, A Guide to Methods and Applications. M. Innis, D. Gelfand, J. Sninsky, and T. White, eds. Academic Press, San Diego, CA.

51. Woudt, L. P., Pastink, A., Kempers-Veenstra, A. E., Jansen, A. E., Mager, W. H., and Planta, R. J. 1983. The genes coding for histone H3 and H4 in Neurospora crassa are unique and contain intervening sequences. Nucleic Acids Res. 11:5347-5360.

52. Yang, H. A., and Sweetingham, M. W. 1998. The taxonomy of Colletotrichum isolates associated with lupin anthracnose. Aust. J. Agric. Res. 49:1213-1223. 